\title{
SMEH: Stochastic Method of Energy Harvesting for Powering up Mobile Phones
}

\author{
Ruchi Sharma \\ Research Scholar \\ Dept. of Computer Science and Engg. \\ Visvesvaraya Technological University \\ Bangalore, India
}

\author{
S. Balaji, Ph.D. \\ Centre for Emerging Technologies \\ Jain Global Campus, Jain University \\ Jakkasandra Post, Kanakapura Taluk \\ Ramanagara Dist.-562112, India
}

\begin{abstract}
The area of energy harvesting has seen various evolutions among the research community. Although various studies have been attempted to address the issues of energy harvesting in the past decade, but very few studies are focused on using RF energy from mobile phones explicitly. Hence, the prime motive of the proposed study is to showcase a mathematical model of RF energy harvesting using stochastic approach termed as SMEH i.e. Stochastic Method of Energy Harvesting. SMEH is designed analytically and evaluated using simulation based approach considering various near real time constraints of mobile devices, traffic scenario, as well as discrete state definition of the system for better analytical evaluation. The outcome of the proposed system is analyzed using probability for loss of event, queuing delay, throughput and compared with the most recent standard work in the similar direction.
\end{abstract}

\section{Keywords}

RF-Energy harvesting, Stochastic, Probability, Throughput

\section{INTRODUCTION}

The proliferation of RF energy is presently telecasted from billions of radio transmitters as far and wide as possible, including cell phones, handheld radios, versatile base stations, and $\mathrm{TV} /$ radio telecast stations [1]. The capacity to collect RF energy, from surrounding or devoted sources, empowers remote charging of low-power gadgets and has coming about profits to item plan, ease of use, and unwavering quality. Battery-based hardware can be trickled charged to dispense with battery substitution or amplify the working life of frameworks utilizing disposable batteries. Without battery gadgets can be intended to work upon interest or when sufficient charge is amassed. In both cases, these gadgets can be free of connectors, links, and battery access boards, and have flexibility of position and portability amid charging and utilization. The clear offer of collecting encompassing RF vitality is that it is basically "free" source of energy. The quantity of radio transmitters, particularly for portable base stations and handsets, keeps on increasing. Mobile phones speak to an extensive wellspring of transmitters from which to collect RF power, and will possibly empower clients to give power-on-interest for an assortment of short proximity sensing applications. Likewise, consider the quantity of Wi-fi switches and remote end gadgets, for example, laptops. In some urban situations, it is conceivable to truly locate several Wi-fi access focuses from a solitary area. At short go, for example, inside the same room, it is conceivable to collect a small measure of vitality from an average Wi-fi switch transmitting at a force level of 50 to $100 \mathrm{mw}$ [2].

RF power can be telecasted in unlicensed groups, for example, $868 \mathrm{mhz}, 915 \mathrm{mhz}, 2.4 \mathrm{ghz}$, and $5.8 \mathrm{ghz}$ when more power or more unsurprising vitality is required than what is accessible from surrounding sources [3]. At $915 \mathrm{mhz}$, legislature regulations restrain the yield force of radios utilizing unlicensed recurrence groups to $4 \mathrm{w}$ successful isotropic radiated power, as on account of radio-frequency ID investigative specialists. The RF energy can be utilized for charging or operating in a wide range of low-powered devices. It is already known that ambient radio frequencies are universally existing over an maximized range of available frequencies as well as levels of energy. It is more available in urban area populated with users with more number of mobile devices. The RF waves emitted from such mobile devices uniquely represents a widely availability of the power if it can be efficiently used for harvesting the energy for charging the low-powered computing devices. With the growing number of population adopting smart phones and usage of $3 \mathrm{G}$ networks, there is a large availability of RF waves that can be exploiting for the purpose of energy harvesting. Dedicated power transmitters further enable engineered and predictable wireless power solutions. With continued decreases in the power consumption of electronic components, increased sensitivity of passive receivers for RF harvesting, and improved performance of low-leakage energy storage devices, the applications for wire-free charging by means of RF-based wireless power and energy harvesting will continue to grow.

This paper presents a mathematical model that adopts stochastic and probability theory to perform optimization of the RF energy for powering up mobile devices. Section II discusses about some of the recent studied performed in the area of energy harvesting followed by Section III that discusses about the problem identification of the proposed study. Proposed model is briefly introduced in Section IV, while Section V discusses about the adopted design principles. The implementation part of the proposed system is discussed in Section VI, while the result discussion with respect to the comparative performance analysis is discussed in Section VII. Finally, Section VIII makes some concluding statement of the proposed study and summarizes the outcome of the study.

\section{RELATED WORK}

This section discusses about the prior research techniques that have been presented by various authors in research manuscript about energy management of mobile devices.

Hsu et al. [4] have presented a framework of provisioning system to ensure energy management of mobile devices. The study has used dynamic energy management to guarantee effective energy harvesting techniques. The outcome of the study exhibited that the framework can accomplish better neutrality of energy between drained energy as well as harvested energy to ensure optimal operation of energy harvesting and better provisioning system.

Khandaker et al. [5] have performed investigation on the power system for antenna theory. The authors have focused on the 
non-convex optimization issues arising from the constraints of power in harvested energy when the signal is transmitted to the multiple base antennas. The outcome of the study was found to show minimal complexity using zero-forcing transmit beamforming techniques.

Lemey et al. [6] have presented their study that has integrated energy management components with energy harvesting hardware (transducers) as well as a storage component on a pre-determined antenna topology. The study has provided a significant guideline to guarantee lesser dependency of hardware towards energy scavenging mechanism on antenna's performance.

The studies toward scheduling as well as provisioning problem was investigated by Chetto et al. [7], as it is directly associated with the drainage of energy supply. The author commented that if the scheduling is not performed effectively, the storage module depletes unnecessary power to perform a task. Hence, the authors have introduced a scheduling algorithm termed as Earliest Deadline First (EDF) that is considered both for load balancing as well as for real-time task scheduling process.

Shigeta et al. [8] have introduced a framework for optimizing the sensing rate of sensor nodes by using RF energy harvesting principles. The authors have used energy dissipation control policies based on duty cycle for maximizing energy to be harvested from RF. The prime problem addressed in this paper is the energy leakage problem which is rarely found as a matter of issues to other research topic in energy harvesting.

Some of the significant studies towards energy harvesting were witnessed even in the field of medical science. Ouda et al. [9] have introduced an implantable intra-ocular pressure monitoring system by using an energy harvester of $5.2 \mathrm{GHz}$ power. It was studied that the design principle of the energy harvester has been accomplished over on-chip antenna for the purpose of receiving both data as well as RF energy. The prototype was tested in real-time to ensure the outcomes. Le et al. [10] have introduced a framework that can potentially transform energy of far-field RF to DC voltage at a very minimal received energy as well as voltage. The authors have designed this prototype considering 36-stage rectifier and CMOS technology to ensure efficient storage of power.

Various authors were found to persistently discuss about the usage of RF energy harvesting system considering from cell towards. One of such notable author will be Arrawatia et al. [11], who have designed antenna based on maximized gain electromagnetic design. The complete design principle for energy harvesting was done in real-time. Another author Olivo et al. [12] have spoken about the possible scopes and limitations of the existing energy harvesting principles. The study was theoretically sound as it discusses about the inductive links associated with the power consumption of wireless connectivity. The author have discussed that energy storage device dissipate power depending upon the dynamic usage of the consumers.

A remarkable study was performed by Gummeson et al. [13] who has studied about the energy harvesting from the core design principles. The authors have discussed the strategies of micro-harvesting formulated by them to enable the maximized efficiency in energy performance. The outcome of the studies was found to be benchmarked using trace-driven simulator for performing modelling as well as analyzing the outcomes. The outcomes of the study were tested on multiple mobility condition with variations in simulation parameters in energy harvesting.
The study presented by Gilbert et al. [14] has discussed various potential factors of energy requirements of sensor nodes in conventional wireless sensor network. The framework used in the study have successfully evaluated source of ambient energy and discussed about the extensive charecteristics of a wide scope of energy transformation equipments. The uniqueness in this study is its framework that compares various availability of ambient resources and performs transformation mechanism in terms of normalized density energy.

It was also seen that majority of the existing studies towards energy efficiency and energy harvesting has been studied from Rectenna designs. Research in similar direction was carried out by Ahmad et al. [15] who have proposed a new prototype for Rectenna. The prototype design of Rectenna uses FM waves and adopts the design principle of fractal antenna. The outcome of the study has shown better effective strategy for extracting necessary power for domestic utility.

Mahalakshmi et al. [16] have discussed an approach for feed implantable system. The design principle of the study is almost similar to work of Ouda et al. [9]. In this principle, the circuit is designed for energy harvesting for the purpose of supplying energy to the implantable medical devices using wireless medium. The study has adopted thermoelectric generator for harvesting energy extracted from human body.

Beeby et al. [17] have reviewed some of the techniques where by adopting vibration factor is used for energy harvesting. The study has illustrated various self-powered micro-systems as well as other electrical systems where energy plays a critical role in their respective operation. The study have also discussed various inherent mathematical theories for inertialbased generators with various challenging scenarios to testify the effectiveness of energy storage and distribution after harvesting is performed.

\section{PROBLEM IDENTIFICATION}

Our prior study [18] have discussed majority of the significant studies done in the area of energy harvesting and discussed about their loopholes. It was found that one of the biggest problems in energy harvesting is obtaining the correct sources of energy which are required to be cost effective, available, reliable, and consistent. In the area of wireless networking system, the deployment of energy harvesting methods is eventually encountering various limitations as well as constraints for ensuring the effectiveness of the energy harvesting equipment. The energy requirements of the wireless systems provided by the traditional battery will require conserving the harvested energy for further operations. Hence, there is an exponential need for designing an energy harvesting device that can provide abundant energy for wireless systems in an efficient manner. The research towards harnessing the existing RF sources dated a decade old only. The prime purpose of this exploration is to power up the low-powered devices from the ambient RF energy sources like signals captured from mobile phones or equivalent communicating devices. However, the existing RF levels are found to be highly minimal than those provided by a dedicated RF levels. Hence, it poses a significant research challenge in maximizing the efficiency of the energy harvesting system even for low-powered devices. It has been observed that from the year 2011 to 2014, there has been introduction of an exclusively techniques that addresses the issues of RF energy harvesting. The research gaps explored from the studies are as follows:

- There are very less work being explored for addressing RF energy harvesting exclusively for mobile device, where majority of the studies are more or less concentrated on 
low powered device like sensors. Studies towards powering up mobile phone are few to find.

- The majority of the studies presented till date [18] are addressing the issues of energy harvesting using various techniques, however, none of the techniques are found to efficiently being benchmarked which cause a bit uncertainty in adopting such framework for future usage.

Hence, the proposed scheme introduces a stochastic modelling for accomplishing the objective of the harvesting RF energy for the mobile device that runs effectively on the mobile device itself. The principle design concept, therefore, must include various real-time constraints of event loss probability as well as delay for the purpose of understanding the effectiveness in the design formulation. In order to further strengthen this formulation, the proposed system performs mathematical modelling of various condition required to ensure better harvesting scheme, where optimization will play a very crucial role. It is believed that proposed system will significant overcome the research gap discussed in our prior work [18].

\section{SMEH: STOCHASTIC MODEL FOR ENERGY HARVESTING}

The prime purpose of the proposed study is to develop a stochastic model for developing RF energy harvesting using mobile devices (or nodes) that can integrate the process of energy harvesting, event arrivals, energy storage, and queuing unprocessed events. The study has coined the proposed model as SMEH i.e. Stochastic Model for Energy Harvesting exclusively for the mobile device. The schema of the proposed system is exhibited in Fig. 1 and Table 1 shows the notations that are used in the design principle of the proposed SMEH.

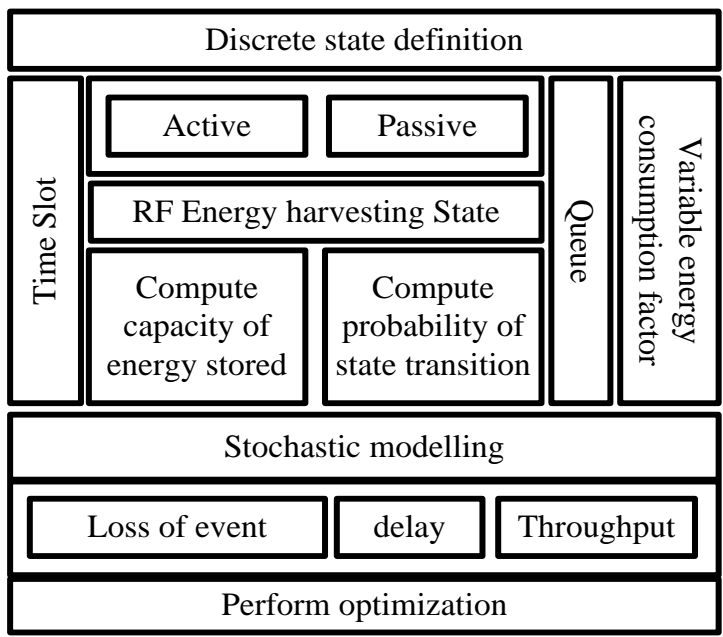

Fig 1: Proposed Schema of SMEH

Table 1: Notation Used

\begin{tabular}{|l|l|}
\hline $\mathrm{r}$ & rate \\
\hline$\sigma_{\mathrm{a}}$ & Energy harvesting parameter for active to passive state \\
\hline$\sigma_{\mathrm{p}}$ & Energy harvesting parameter for passive to active state \\
\hline $\mathrm{C}$ & Channel capacity \\
\hline $\mathrm{E}$ & Capacity of queue \\
\hline$\eta$ & Number of events \\
\hline $\mathrm{P}$ & Power \\
\hline
\end{tabular}

\begin{tabular}{|l|l|}
\hline$T_{d}$ & Discrete time \\
\hline $\operatorname{Pr}_{\mathrm{a}, \mathrm{p}}$ & Transition probability from active to passive state \\
\hline $\operatorname{Pr}_{\mathrm{p}, \mathrm{a}}$ & Transition probability from passive to active state \\
\hline $\mathrm{T}_{\mathrm{s}}$ & Time slot \\
\hline $\mathrm{x}$ & Stored energy matrix \\
\hline$\lambda$ & Queue \\
\hline$\delta$ & Delay \\
\hline $\operatorname{Pr}_{\mathrm{t}}$ & Probability of event loss \\
\hline$\Delta$ & Energy efficiency parameter \\
\hline$\theta_{1}$ & Parameter for energy efficiency for energy storage \\
\hline$\theta_{2}$ & Parameter for buffer \\
\hline$\theta_{3}$ & Parameter for power harvester \\
\hline
\end{tabular}

\section{SMEH: DESIGN PRINCIPLES}

The formulation of proposed SMEH is completely based on mathematical backgrounds using probability theory. For this purpose, the proposed SMEH considers to be working in active and passive harvesting source. The active state is defined as a state when the mobile nodes perform computation of the energy at a particular rate whereas passive state represents a state with zero computation of energy. As the proposed system uses stochastic principle, so the time factor for active and passive states can be represented as $\sigma a$ and $\sigma p$ respectively for exponential independent distribution. The system also considers a rechargeable unit as energy storage system for the mobile device with certain capacity $\mathrm{C}$. The proposed design considers that there is no energy seepage at any point of energy storing process. The prime design principle of the SMEH is depicting energy depletion as set of events that includes data sensing as well as processing. The proposed system also considers the incoming events to be arranged in FCFS queue with a buffer to store $\mathrm{E}$ events. The event mapping principle of the SMEH is based on the fact that event is a representation of set of observations, estimates, and aggregated data with references to the mobile applications. For easiness in computation, the proposed system considers a constant amount of data being generated by every events to be received, transmitted and processed and hence it depletes a specific amount of power from the mobile device.

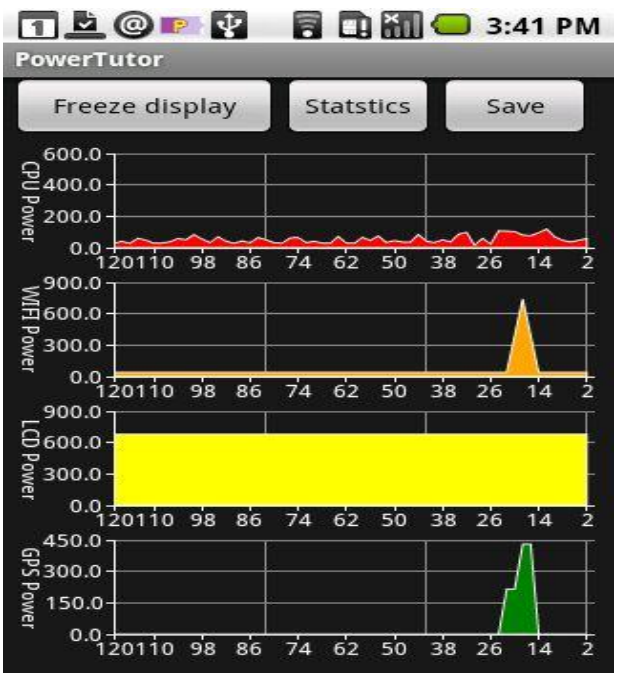

Fig 2: Energy Consumption in Android Phone 
As shown in Fig.2, the mobile device has various apps (CPU, WiFi, LCD, GPS, etc) that have its own rate of energy consumption. For an effective processing of every event, an adequate range of power is required in the mobile battery. Hence, the capacity of energy storage (battery) $\mathrm{C}$ can be defined as,

$$
\mathrm{C}=\eta . \mathrm{P}
$$

In the above eq (1), $\eta$ is the vector representing number of events, while P represents amount of power being in used. The proposed system considers that energy required for event processing is instantly removed from the energy storage when there is adequate power. In the initial stage of design, the time factor is discretized and is represented as $T_{d}$, thereby, it converges the probability model with discrete time for evolving up with stochastic modelling.

Hence, if $\mathrm{T}_{\mathrm{d}}<<1$, than probability factor (harvesting state-1: $\sigma_{\mathrm{a} .} \mathrm{T}_{\mathrm{d}}$ ), (harvesting state-2: $\left.\sigma_{\mathrm{p}} \mathrm{T}_{\mathrm{d}}\right)<<1$ is very high. Hence, the two states performs transition only once at maximum during $\mathrm{T}_{\mathrm{d}}$. Therefore, the probability of transition from active to passive state is,

$$
\operatorname{Pr}_{a, p}=\sigma_{a} T_{d} \cdot e^{-\sigma_{a} \cdot T_{d}} \approx \sigma_{a} T_{d}
$$

And the probability of transition from passive to active to state

$$
\operatorname{Pr}_{p, a}=\sigma_{p} T_{d} \cdot e^{-\sigma_{p} \cdot T_{d}} \approx \sigma_{p} \cdot T_{d}
$$

Just like eq.(2) and eq.(3), in case of no events less than or equal to $1\left(\left(\sigma_{\mathrm{e}} \mathrm{T}_{\mathrm{d}}\right)<<1\right)$ during $\mathrm{T}_{\mathrm{d}}$ can be presented by probability factor

$$
\operatorname{Pr}=\sigma_{e} T_{d} \cdot e^{-\sigma_{e} \cdot T_{d}} \approx \sigma_{e} \cdot T_{d}
$$

The mathematical modelling principle also hypothesize that a mobile node will always require to stay in active state to perform harvesting of energy to be measured in a unique time slot defined as

$$
T_{s}=\frac{P}{r \cdot T_{d}}
$$

Therefore, substituting contents of $P$ from eq.(5) in eq.(1), the mathematical representation can be now modified as,

$$
\mathrm{C}=\eta \cdot \mathrm{T}_{\mathrm{s}} \cdot \mathrm{r} \cdot \mathrm{T}_{\mathrm{d}}
$$

Hence, in order to perform energy harvesting, the storage device can stand upto $\eta T_{s}$ units of energy, where a unit of energy can be represented as

$$
r . T_{d}=\frac{P}{T_{s}}
$$

Therefore, the cumulative state of the energy harvesting for the considered mobile device can be computed by the i) number of the events organized in queue, ii) residual energy in the storage form, and iii) state of harvesting. According to the consideration of the proposed stochastic model, the length of the queue will be non-zero $(0<$ queue $<1)$ for the condition stored energy $<\mathrm{P}$, as it is known that only with availability of adequate power, events can be processed. Hence, with these conditions, it can be stated that events that are waiting in the queue is downbeat stored energy. Therefore the model evaluates its state space variables as $\left\{(\mathrm{x}, \mathrm{s}) \mid \mathrm{x} \in\left\{\mathrm{T}_{\mathrm{s}} . \mathrm{E}, \ldots . . \mathrm{T}_{\mathrm{s}} . \eta\right\}\right\}$ and $\operatorname{se}(0,1)\}$. The formulation of the state space variables considers factor $x$ as the energy being stored. Therefore, the actual mathematical representation of the energy stored as well as the quantity of the events being gathered in queue system can be shown as,

$$
P(x)=\left\{\begin{array}{cc}
\frac{x}{T_{s}} \cdot P & x \geq 0 \\
\left(\frac{x}{T_{s}}+\left(-\frac{x}{T_{s}}\right)\right) \cdot P & x<0
\end{array}\right.
$$

And

$$
\lambda(x)=\left\{\begin{array}{cc}
0 & x \geq 0 \\
\left(-\frac{x}{T_{s}}\right) & x<0
\end{array}\right.
$$

The system considers active and passive harvesting states as $\mathrm{s}=1$ and $\mathrm{s}=0$ respectively. However, for illustrative computation, the states are re-formulated as $i=2\left(x+T_{s} \cdot E\right)+s$ in the form of matrix.

Considering the transition probability from $\mathrm{i}^{\text {th }}$ state to $\mathrm{j}^{\text {th }}$ state in stochastic modelling, the system identified following set of transition as i) with probability (1-Prap $)$ the mobile node will resume in its state of active, while it will retain in passive state with $\operatorname{Pr}_{\text {ap }}$ probability, ii) with probability $\left(1-\mathrm{Pr}_{\mathrm{pa}}\right)$, the mobile node will resume in its state of passive, while it will retain in active state with $\operatorname{Pr}_{\text {pa }}$ probability, iii) unless the energy is adequate, in case of active state, the mobile node gains one unit of energy, iv) in case of no event, the mobile nodes loses $\mathrm{T}_{s}$ energy units with $\mathrm{P}_{\mathrm{r}}$ probability. The simpler representation of this mathematical modelling for passive state can be done as,

$$
\operatorname{Pr}_{i<2 T_{s}, j}=\left\{\begin{array}{cc}
1-\operatorname{Pr}_{p, a} & j=i \\
\operatorname{Pr}_{p, a} & j=i+1 \\
0 & \text { otherwise }
\end{array}\right.
$$

And

$$
\operatorname{Pr}_{i \geq 2 T_{s}, j}=\left\{\begin{array}{cc}
\left(1-\operatorname{Pr}_{p, a}\right)(1-\operatorname{Pr}) & j=i \\
\left(1-\operatorname{Pr}_{p, a}\right) \operatorname{Pr} & j=i-2 T_{s} \\
\operatorname{Pr}_{p, a}(1-\operatorname{Pr}) & j=i+1 \\
\operatorname{Pr}_{p, a} \cdot \operatorname{Pr} & j=1-2 T_{s}+1 \\
0 & \text { otherwise }
\end{array}\right.
$$

The simpler representation of mathematical modelling for active states can be done as,

$\operatorname{Pr}_{i<2 T_{s}, j}=\left\{\begin{array}{cc}1-\operatorname{Pr}_{a, p} & j=i+2 \\ \operatorname{Pr}_{a, p} & j=i+1 \\ 0 & \text { otherwise }\end{array}\right.$

And

$$
\operatorname{Pr}_{2 T_{s}-1 \leq i \leq 2 T_{s}(\eta+E), j}=\left\{\begin{array}{cc}
\left(1-\operatorname{Pr}_{a, p}\right)(1-\operatorname{Pr}) j=i-2 T_{s}+2 \\
\left(1-\operatorname{Pr}_{a, p}\right) \operatorname{Pr} & j=i+2 \\
\left.\operatorname{Pr}_{a, p} . \operatorname{Pr}\right) & j=i-2 T_{s}+1 \\
\operatorname{Pr}_{a, p} \cdot(1-\operatorname{Pr}) & j=i+1 \\
0 & \text { otherwise }
\end{array}\right.
$$




$$
\operatorname{Pr}_{i=2 T_{s}(\eta+E)+1, j}=\left\{\begin{array}{cc}
\left(1-\operatorname{Pr}_{a, p}\right) \operatorname{Pr} & j=i-2 T_{s}+2 \\
\left(1-\operatorname{Pr}_{a, p}\right)(1-\operatorname{Pr}) & j=i \\
\operatorname{Pr}_{a, p} \cdot \operatorname{Pr} & j=i-2 T_{s}+1 \\
\operatorname{Pr}_{a, p} \cdot(1-\operatorname{Pr}) & i=i-1 \\
0 & \text { otherwise }
\end{array}\right.
$$

\section{IMPLEMENTATION}

The proposed system is implemented on 32 bit windows operating, where the emphasize was initially laid on the correctness of the mathematical formulations. The proposed system is evaluated using the mean delay $(\delta)$ and likelihood of event loss $\left(\mathrm{Pr}_{\mathrm{t}}\right)$. The system considers that the parameters responsible for harvesting $\left(\sigma_{\mathrm{p}}\right.$, and $\left.\sigma_{\mathrm{p}}\right)$ always depends on the properties of the energy source, where the event arrival rate always depends on the extent of the mobile application used by the user in 24 Hours. Therefore, the algorithm implementation of the proposed SMEH is done by considering the critical parameters like harvesting energy ( $r$, size of the harvester), capacity of queue (E), and capacity of energy storage device (१). The system also formulates energy efficiency factor to understand the extent of the energy that it preserves and uses in the absence of complete depletion of energy from the mobile battery. Therefore, for this reason, the variable energy consumption factor is coined that can be mathematically expressed as,

$$
\Delta(\eta, E, r)=\theta_{1} \cdot \eta+\theta_{2} \cdot E+\theta_{3} \cdot r
$$

The above equation shows that the energy efficiency is computed using $\theta_{1}, \theta_{2}$, and $\theta_{3}$ that represent unit charge for energy efficiency for energy storage, buffer, and power harvester respectively. Therefore, now, the problem represented by the above equation should be subjected to minimization for better optimization of energy efficiency. Hence, it can be now represented as,

$$
f(x)_{\min }=\theta_{1} \cdot \eta+\theta_{2} \cdot E+\theta_{3} \cdot r
$$

The prime objective of eq. (13) is to perform minimization to solve variable optimization issues. The computation of the proposed system is iterated till it finds the best minimization points done using probability theory and stochastic principle where the delay constraint should be reduced as far as possible. The implementation of the proposed system is done considering the likelihood of the event loss as a function of $(\eta+E)$. The formulation is done in this way as it is the only solution to ensure that algorithm can process the energy factor of storage as well as optimized capacity of the queue without any significant effect on the likelihood of the event loss. Hence, better outcome of the energy to be harvested can be ensured. The approximated mean delay in this process is computed using standard Little's formula [19]. The algorithm that was implemented for the purpose of designing proposed SMEH is as exhibited below:

\begin{tabular}{l} 
Algorithm for SMEH \\
Input: Harvesting states, energy variables, node parameters \\
Output: Optimized Harvested Energy \\
Start \\
1. Define harvesting states $\left(\sigma_{\mathrm{a}} \sigma_{\mathrm{p}}\right)$ \\
2. Compute capacity of energy storage \\
$\qquad \mathrm{C}=\eta \cdot \mathrm{P}=\eta \cdot \mathrm{T}_{\mathrm{s}} \cdot \mathrm{r} \cdot \mathrm{T}_{\mathrm{d}}$ \\
\hline
\end{tabular}

$$
\begin{aligned}
& \text { 3. Evaluate probability of transition } \\
& \qquad \begin{array}{l}
\operatorname{Pr}_{a, p}=\sigma_{a} T_{d} \cdot e^{-\sigma_{a} \cdot T_{d}} \approx \sigma_{a} T_{d} \\
\operatorname{Pr}_{p, a}=\sigma_{p} T_{d} \cdot e^{-\sigma_{p} \cdot T_{d}} \approx \sigma_{p} \cdot T_{d} \\
\operatorname{Pr}=\sigma_{e} T_{d} \cdot e^{-\sigma_{e} \cdot T_{d}} \approx \sigma_{e} \cdot T_{d}
\end{array}
\end{aligned}
$$

4. Compute Probability for passive state (Eq.(10)

5. Compute Probability for active state (Eq.(11)

6. Performing Optimization:

$$
f(x)_{\min }=\theta_{1} \cdot \eta+\theta_{2} \cdot E+\theta_{3} \cdot r
$$

7. Compute likelihood of event loss owing to queue overflow

8. Compute proportion of mean energy harvested to the mean required power

$$
H_{p}=\frac{\sigma_{p} \cdot r}{\left(\sigma_{a}+\sigma_{p}\right) \cdot \sigma_{e} \cdot P}
$$

9. Evaluate likelihood of packet loss

$$
\lim _{\eta \rightarrow \infty o r E \rightarrow \infty} \operatorname{Pr}=\left\{\begin{array}{cc}
1-H_{p} & H_{p}<1 \\
0 & H_{p}>1
\end{array}\right.
$$

10. Compute mean delay

11. Go to 6 .

End

The above algorithm also exhibits in case the mobile node is under-resourced, the mean delay will be non-zero element while if the mobile node is over-resourced, the stored energy will be maximized to larger bound. Therefore, for better optimization, it is essential that queue should be adequately large as it can significant minimize the delay.

\section{RESULT ANALYSIS}

The implementation of the proposed system is evaluated using the certain performance parameters like extent of energy being harvested, approximated average delay, probability of event loss, average connection arrival rate, and throughout with respect to the number of harvester nodes. For effective analysis of the outcome of the study, we selected the recent work performed by Hoang et al. [20]. The prime reason of selecting this particular work is because i) this is the most recent study done in the line of energy harvesting and ii) the techniques adopted by Hoang et al. [20] is also based on probability theory. The authors have presented optimization technique for performing channel access to harvest RF energy using learning algorithm. Markov decision process is the backbone of the study performed by Hoang et al. [20], where the prime intention was to basically maximize the throughput of the channel. The technique adopted is definitely the best of its solution till date in the area of energy harvesting, as authors [20] have introduced an online learning algorithm, which the help of which the secondary users can obtain channel access policy. 


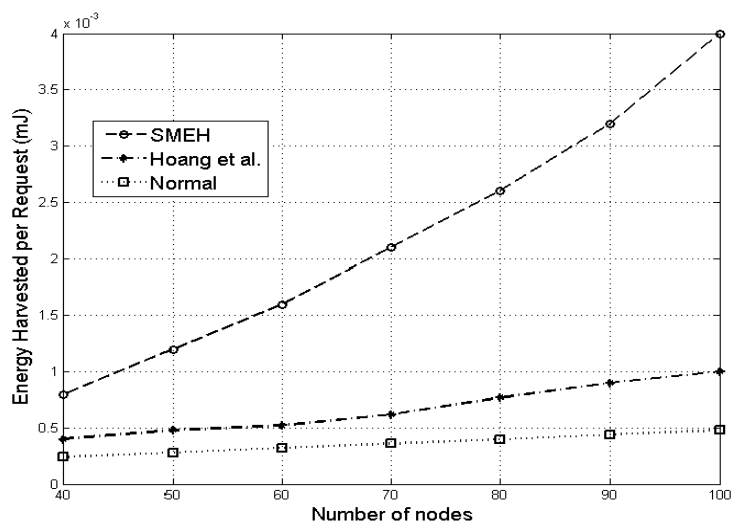

Fig 3: Energy harvested per request

Fig.3 highlights the comparative performance analysis of the extent of the energy being harvest per request for the user considered in increasing number of harvester nodes. The outcome of the study shows that under normal circumstances, the extent of energy harvesting is quite a challenging problem owing to dynamic scenario of the traffic. As such smart optimization of energy usage principle is missing in the normal mobile devices, so the curve representing energy harvesting under normal mode is found to be quite less. Hoang et al.[ 20] process was found to be little better than normal mode owing to the learning algorithm, as it can adapt itself with different kinds of dynamic scenario due to Markov modelling techniques. However, implementation of [20] didn't discretely discuss about the state of transition, for which reason, it misses to calculate the extent of energy to be harvested for mobile device. In the proposed SMEH, we perform discrete modelling of the states and apply our algorithm based on those states (active and passive), for which reason, the optimization principle formulated can have better results.

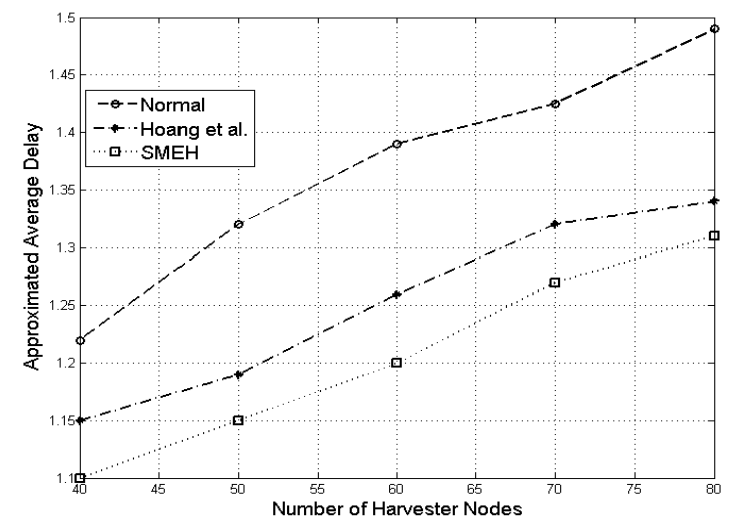

Fig 4: Approximated Average Delay

Fig.4 represents the approximated average delay of the outcomes of the study with respect to number of harvester nodes. The outcome shows that under normal condition, the average queuing delay is quite high owing to the absence of queue management. Hoang et al. [20] have used normal queue which is used only by the secondary users for transmitting the data packet, where the queue was found to be static. However, the proposed system considers dynamic queue management based on the likelihood of event loss, which is quite decreasing in nature. For this reason, the proposed system excels better compared to the existing system owing to less computational complexity of the algorithm and therefore exhibits better delay performance with increasing number of harvester nodes.

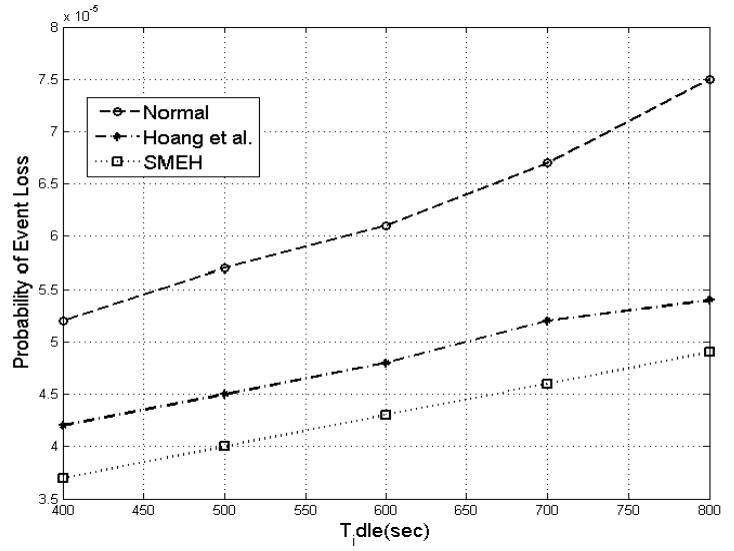

Fig 5: Probability of Event Loss

Fig.5 highlights the comparative performance evaluation for probability of event loss with respect to Time. For this outcome evaluation, we consider a third state called as idle state, which will mean that a mobile device will be able to perform accessing channel. We denote it by $\mathrm{T}_{\text {idle }}$ in seconds that basically represents time period in idle mode(or state). The proposed system, in the initial phase of algorithm, was found to have slower transition of harvesting mode from active to passive states. In the proposed system, with the same value of harvesting parameter $\sigma_{\mathrm{p}}$, it was found that larger $\sigma_{\mathrm{a}}$ is obtained, for which reason, the balancing became easier. The system persistent attempted to consume less energy at the time of energy harvesting for better outcomes. The mean delay for queue can converge to a fixed point when the queue $\mathrm{E}$ is relatively full for larger value of $\sigma_{\mathrm{e}}$. However, it was also attempted that mobile device should harvest optimally, but cases can occur, when the extent of energy harvested is quite more than it consumes. Hence, it can be said that maximized likelihood of loss of event basically corresponds to the maximal mean delay in majority of the simulation scenarios. The likelihood of loss is quite equivalent (or close) to 1 at higher value of $\sigma_{e}$, this will eventually mean that majority of the events are missed as an alternative of arranging in queue structure. Therefore, probability of event loss is quite less for the proposed SMEH, whereas for the existing approach, it is quite high. Another reason for this performance is the optimization principle that always tries to minimize the cost of energy consumption at the time of energy harvesting. Hence, better equilibrium is maintained between harvesting and energy consumption for better outcomes of system as well as the network.

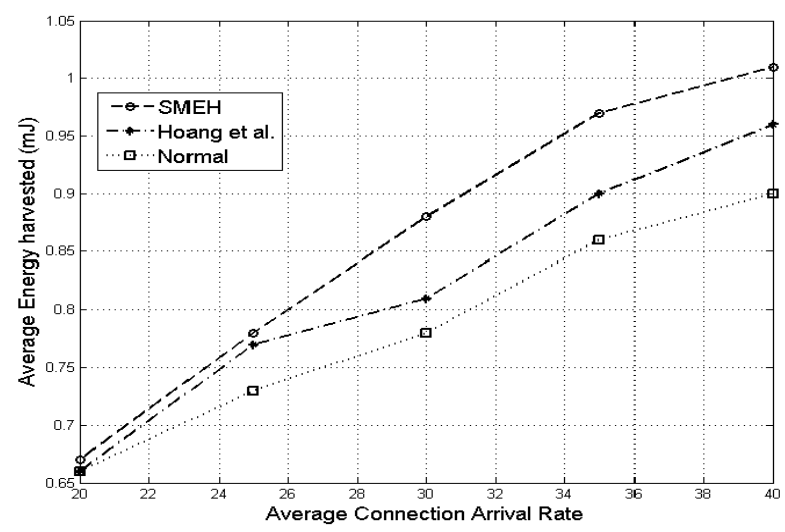

Fig 6: Average Energy Harvesting 
Fig.6 represent outcome of the proposed study that highlights the average energy harvesting with the increasing number of average connection arrival rate. It is said that when the mobile device uses $3 \mathrm{G}$ or $4 \mathrm{G}$ network, the energy consumption of the device is quite high. We choose to perform this experiment analytically, as it is quite obvious that when a mobile device uses $3 \mathrm{G} / 4 \mathrm{G}$ connectivity, there are multiple apps that attempts to download/upload the data packet massively and all simultaneously resulting in faster depletion of energy. In such condition, even if the harvester node is present, the system might force the harvester node to storage higher quantity of energy, which will degrade the system performance. Hence, the outcome shows that with the better policies of channel access, reduced loss events, the system is able to harvest more quantity of energy for storage.

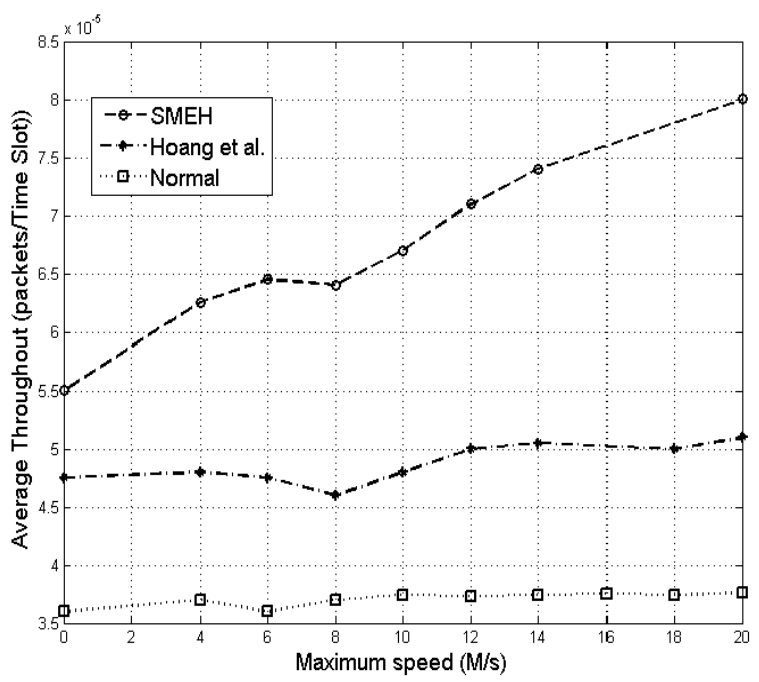

Fig 7: Average Throughput

Fig.7 shows the outcome of the average throughout with the increasing number of speed of the user (node). Mobility plays a crucial role in throughput. If the mobility is high, it is expected that throughput may be slightly effected and may result in power outage at one time. Hence, the simulation study is performed by analytically enhancing the speed in meter per seconds, where the throughout is evaluated using packets per time slots. Owing to iterative optimization process, the proposed study shows superior throughout in every sequential time slots, where the mobility doesn't seem to have a significant impact on the performance. However, in Hoang et al. [20], the system is completely dependent on online learning algorithm, which requires a massive amount of information of the nodes. Hence, a higher level of computation is done for which the system could somehow give better energy efficiency performance, but low in throughput performance.

Hence, the proposed system offers better system as well as network performance, which is actually compatible with existing usage of $3 \mathrm{G} / 4 \mathrm{G}$ networks on the run. It also ensures that the harvesting is done effectively and optimally, by exploiting the discrete state charecteristics of the mobile nodes.

\section{CONCLUSION}

The present paper discusses about a mathematical model that uses stochastic theory to perform energy harvesting from RF mobile device. The uniqueness of the proposed system is that it offers a very simple design of probabilistic logic, where various real-time scenarios of energy harvesting as well as energy consumption is addressed and their challenging conditions are considered. The proposed system is completely designed analytically and studied based on simulation being performed using Matlab. The outcome of the study is scrutinized deeply and compared with the most recent work done to find that proposed system has better performance and better sustainance capability of energy harvesting for long terms. Our work towards future direction will be focused on enhancing the present model considering multiple sources for further analysis of event loss owing to energy outage. We will also consider ambient resources and perform analysis of test-environment using multiple sophisticated test environments to ensure that our techniques and method can assist the streamline flow of energy to the mobile device in odd hours and odd situation, where existing energy harvesting techniques fails. We will also consider various real-time scenarios like unavailability of RF harvester node in the future studies for evolving up with some unique outcomes.

\section{REFERENCES}

[1] S. Priya, D. J. Inman, "Energy Harvesting Technologies", Springer Science \& Business Media, Technology \& Engineering - 544 pages, 2008

[2] S.Shrestha, S-K Noh, D-Y Choi, "Comparative Study of Antenna Designs for RF Energy Harvesting", International Journal of Antennas and Propagation, Article ID 385260, 10 pages, http://dx.doi.org/10.1155/2013/385260, 2013

[3] http://www.mouser.in/applications/rf_energy_harvesting/

[4] R.C.Hsu, C-T. Liu, H-L.Wang, "A Reinforcement Learning-Based ToD Provisioning Dynamic Power Management for Sustainable Operation of Energy Harvesting Wireless Sensor Node," IEEE Transaction on Emerging Topics in Computing, Vol.2, No.2, pp.181191,2014

[5] M.R.A.Khandaker and K-K. Wong, "Masked Beamforming in the Presence of Energy-Harvesting Eavesdroppers", IEEE Transactions on Information Forensics and Security, 2013

[6] S. Lemey, F.Declercq and H. Rogier, "Textile Antennas as Hybrid Energy-Harvesting Platforms", Proceedings of the IEEE, Vol. 102, No. 11, 2014

[7] M. Chetto, "Optimal Scheduling for Real-Time Jobs in Energy Harvesting Computing Systems", IEEE Transactions on Emerging Topics in Computing, Vol.2, No.2, pp.122-133, 2014

[8] R. Shigeta, T. Sasaki, D.M. Quan, “Ambient-RF-EnergyHarvesting Sensor Device with Capacitor-Leakage-Aware Duty Cycle Control", IEEE Transaction on Sensor Journals, 2013

[9] M.H.Ouda, M.Arsalan, L. Marnat, A. Shamim, and K. N. Salama, "5.2-GHz RF Power Harvester in 0.18-/spl mu/m CMOS for Implantable Intraocular Pressure Monitoring", IEEE Transactions on Microwave Theory and Techniques, Vol.61, No. 5, pp.2177-2184, 2013

[10] T. Le, K. Mayaram, and T. Fiez, "Efficient Far-Field Radio Frequency Energy Harvesting for Passively Powered Sensor Networks", IEEE Journal of Solid-State Circuits, Vol. 43, No. 5, 2008

[11] M. Arrawatia, M. S. Baghini and G. Kumar, "RF energy harvesting system from cell towers in $900 \mathrm{MHz}$ band", IEEE-Conference In Communications, pp. 1-5, 2011 
[12] J. Olivo, S. Carrara, and G.D.Micheli, "Energy Harvesting and Remote Powering for Implantable Biosensors", IEEE Sensors Journal, Vol. 11, No. 7, 2011

[13] J. Gummeson, S.S.Clark, K. Fu, and D.Ganesan, "On the limits of effective hybrid micro-energy harvesting on mobile crfid sensors", Proceedings of the 8th international conference on Mobile systems, applications, and services, pp. $195-208,2010$

[14] J.M.Gilbert and F. Balouchi, "Comparison of energy harvesting systems for wireless sensor networks", International Journal of Automation and Computing, Vol.5, No. 4, pp. 334-347, 2008

[15] M.E-d. Ahmad, "Energy Harvesting Using a Cheap Easyto-Fabricate FM Rectenna", In The World Congress on Electronics and Electrical Engineering WCEEENG, Vol. 9, pp. 6-9, 2009

[16] P.Mahalakshmi and S.Kalaiselvi, "Energy Harvesting From Human Body Using Thermoelectric Generator", International Journal of Advanced Research in Electrical,
Electronics and Instrumentation Engineering, Vol. 3, Issue 5,2014

[17] S.P. Beeby, M. J. Tudor and N. M. White, "Energy harvesting vibration sources for microsystems applications", Measurement science and Technology, Vol.17, No. 12, 2006

[18] R. Sharma, S. Balaji, "Investigating Techniques And Research Trends In RF energy harvesting", International Journal of Computer Engineering and Technology, Vol.5, Iss.7, pp. 157-169, 2014

[19] L. Lipsky, "Queueing Theory: A Linear Algebraic Approach", Springer Science \& Business Media, Mathematics - 576 pages, 2008

[20] D. T. Hoang, D. Niyato, P. Wang, D. I. Kim, "Opportunistic Channel Access and RF Energy Harvesting in Cognitive Radio Networks", IEEE Journal on Selected Areas in Communications, Vol. 32, No. 11, 2014 